\title{
MECHANISM OF THE PIVOT SHIFT
}

\author{
H. MATSUMOTO
}

From Keio University, Japan

The mechanism of the pivot shift was investigated by analysing movements under valgus torque in 29 fresh cadaveric knees. The movements were measured in three dimensions, using biplanar photography, when all the ligaments were intact, and then after the ligaments were sequentially divided.

When only the anterior cruciate ligament was sectioned, the pivot shift occurred in seven out of 20 knees examined. In the other 13, though the pivot shift was not observed, an abnormal internal rotation occurred at between $10^{\circ}$ and $50^{\circ}$ of flexion.

Division of the iliotibial tract in addition to division of the anterior cruciate ligament stopped the pivot shift, as the tibia remained internally rotated throughout the range of flexion.

The axis of rotation of the pivot shift was located at the medial collateral ligament, which was kept tight by the applied valgus torque.

The sudden movement in the pivot shift was caused by a complex interaction between the geometry of the knee and the valgus torque applied.

The pivot shift is a characteristic instability of the knee which is commonly observed in knees with ligamentous injuries. Despite the fact that it has been repeatedly studied, both clinically and experimentally (Galway, Beaupré and MacIntosh 1972; Wood and Dandy 1979; Lipke et al 1981; Matsumoto 1987), there is still controversy about its mechanism and cause, the site of the axis about which the movement takes place, and the cause of the sudden movement.

Since the pivot shift is a phenomenon which occurs when a valgus torque is applied, it is important to analyse the movement of the knee under a similar load to investigate the mechanism. Quantitative measurements of knee movement have been carried out previously both on patients and on cadaveric knees (Markolf, GraffRadford and Amstutz 1978; Shoemaker and Markolf 1982), but there have been no studies which measure knee movements under a valgus torque.

\section{MATERIALS AND METHODS}

Twenty-nine knees from fresh frozen cadavers were used; 20 were from men and nine from women, of ages from 22 to 67 years (average 51); there were 24 right and

H. Matsumoto, MD, PhD(Bioeng), Instructor in Orthopaedic Surgery Department of Orthopaedic Surgery, Keio University, 35 Shinanomachi, Shinjuku-ku, Tokyo 160, Japan.

(C) 1990 British Editorial Society of Bone and Joint Surgery 0301-620X/90/5206\$2.00

J Bone Joint Surg [ Br] 1990; 72-B:816-21. five left knees. Those with osteoarthritic changes or injured ligaments were excluded.

An apparatus was constructed to simulate the clinical pivot shift test on a cadaveric knee, and the resulting movements were measured in three dimensions using biplanar photography. The femoral stump of the cadaveric knee was fixed and the tibial stump was held in a socket to which a constant valgus torque could be applied throughout the range of flexion, using a string, pulley and weight system (Fig. 1).

In this preparation the femur was divided at the mid-thigh, so the iliotibial tract (ITT) was detached from its proximal attachments. It was replaced by an artificial ligament with stiffness similar to that of the ITT $(20 \mathrm{~N} /$ m, Fig. 2).

Two wire frameworks, each representing an orthogonal axis system, were attached, one to the femur and one to the tibia (Fig. 3). The joint was then moved from full extension to $90^{\circ}$ of flexion manually under a constant valgus torque (average $12.5 \mathrm{Nm}$ ). The relative movement of the two wire frameworks was recorded by taking a series of biplanar photographs at each $5^{\circ}$ of flexion. After measuring the movement of the normal knee, the ligaments were divided in a predetermined order, and the photographs were repeated. The sequence of experiments in each knee is given in Table I. After all the measurements were completed, the tibia was disarticulated fully from the femur, and several strategically selected points on the femur and the tibia were marked by pins. The relative positions of these points to their respective orthogonal axis systems were measured by 


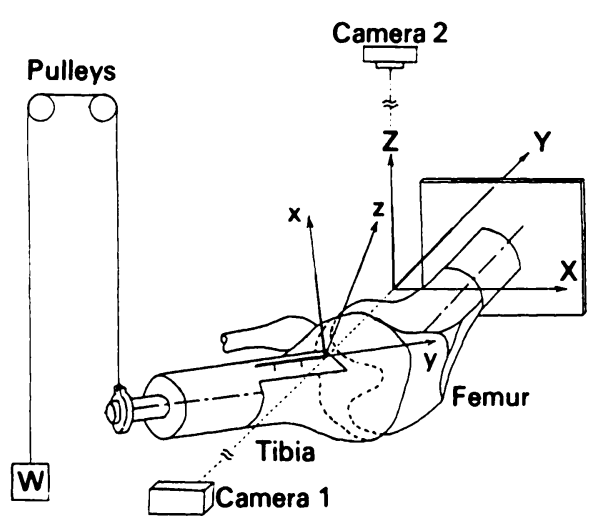

Fig. 1

Figure 1-Schematic drawing of the apparatus to simulate the clinical pivot shift test on a cadaveric knee and to measure its resulting movement in three dimensions, using biplanar photography. Figure 2 - Reproduction of the physiological tension along the iliotibial band with an artificial ligament. Figure 3 - Experimental set-up of the knee and the two wire frameworks, each of which represented an orthogonal axis system.

Table I. Sequence of tests in each joint specimen, by number, and the structure divided

\begin{tabular}{|c|c|c|c|c|}
\hline Specimen & Test 1* & Test 2* & Test 3 & Test 4 \\
\hline $\begin{array}{l}1 \\
2 \\
3 \\
4 \\
5 \\
6 \\
7\end{array}$ & $\begin{array}{l}\text { ACL } \\
\text { Normal } \\
\text { Normal } \\
\text { Normal } \\
\text { Normal } \\
\text { Normal } \\
\text { Normal }\end{array}$ & $\begin{array}{l}\text { ACL } \\
\text { ACL } \\
\text { ACL } \\
\text { ACL } \\
\text { ACL } \\
\text { ACL }\end{array}$ & & \\
\hline $\begin{array}{l}8 \\
9\end{array}$ & $\begin{array}{l}\text { Normal } \\
\text { Normal }\end{array}$ & $\begin{array}{l}\text { ACL } \\
\text { ACL }\end{array}$ & $\begin{array}{l}\text { ITT } \\
\text { ITT }\end{array}$ & \\
\hline $\begin{array}{l}10 \\
11 \\
12\end{array}$ & $\begin{array}{l}\text { Normal } \\
\text { Normal } \\
\text { Normal }\end{array}$ & $\begin{array}{l}\text { ACL } \\
\text { ACL } \\
\text { ACL }\end{array}$ & $\begin{array}{l}\text { Lat cap } \\
\text { Lat cap } \\
\text { Lat cap }\end{array}$ & $\begin{array}{l}\text { ITT } \\
\text { ITT } \\
\text { ITT }\end{array}$ \\
\hline $\begin{array}{l}13 \\
14 \\
15\end{array}$ & $\begin{array}{l}\text { Normal } \\
\text { Normal } \\
\text { Normal }\end{array}$ & $\begin{array}{l}\text { ACL } \\
\text { ACL } \\
\text { ACL }\end{array}$ & $\begin{array}{l}\text { MCL } \\
\text { MCL } \\
\text { MCL }\end{array}$ & \\
\hline $\begin{array}{l}16 \\
17 \\
18 \\
19 \\
20\end{array}$ & $\begin{array}{l}\text { Normal } \\
\text { Normal } \\
\text { Normal } \\
\text { Normal } \\
\text { Normal }\end{array}$ & $\begin{array}{l}\text { ACL } \\
\text { ACL } \\
\text { ACL } \\
\text { ACL } \\
\text { ACL }\end{array}$ & $\begin{array}{l}\text { PCL } \\
\text { PCL } \\
\text { PCL } \\
\text { PCL } \\
\text { PCL }\end{array}$ & \\
\hline $\begin{array}{l}21 \\
22 \\
23\end{array}$ & $\begin{array}{l}\text { Normal } \\
\text { Normal } \\
\text { Normal }\end{array}$ & $\begin{array}{l}\text { Lat cap } \\
\text { Lat cap } \\
\text { Lat cap }\end{array}$ & $\begin{array}{l}\text { ITT } \\
\text { ITT } \\
\text { ITT }\end{array}$ & $\begin{array}{l}\text { ACL } \\
A C L \\
A C L\end{array}$ \\
\hline $\begin{array}{l}24 \\
25 \\
26\end{array}$ & $\begin{array}{l}\text { Normal } \\
\text { Normal } \\
\text { Normal }\end{array}$ & $\begin{array}{l}\text { MCL } \\
\text { MCL } \\
\text { MCL }\end{array}$ & $\begin{array}{l}\text { ACL } \\
\text { ACL } \\
\text { ACL }\end{array}$ & \\
\hline $\begin{array}{l}27 \\
28 \\
29\end{array}$ & $\begin{array}{l}\text { Normal } \\
\text { Normal } \\
\text { Normal }\end{array}$ & $\begin{array}{l}\text { PCL } \\
\text { PCL } \\
\text { PCL }\end{array}$ & $\begin{array}{l}\text { ACL } \\
\text { ACL } \\
\text { ACL }\end{array}$ & \\
\hline
\end{tabular}

-ACL, anterior cruciate ligament; ITT, iliotibial tract; Lat cap, Lateral capsule; MCL, medial collateral ligament; PCL, posterior cruciate ligament

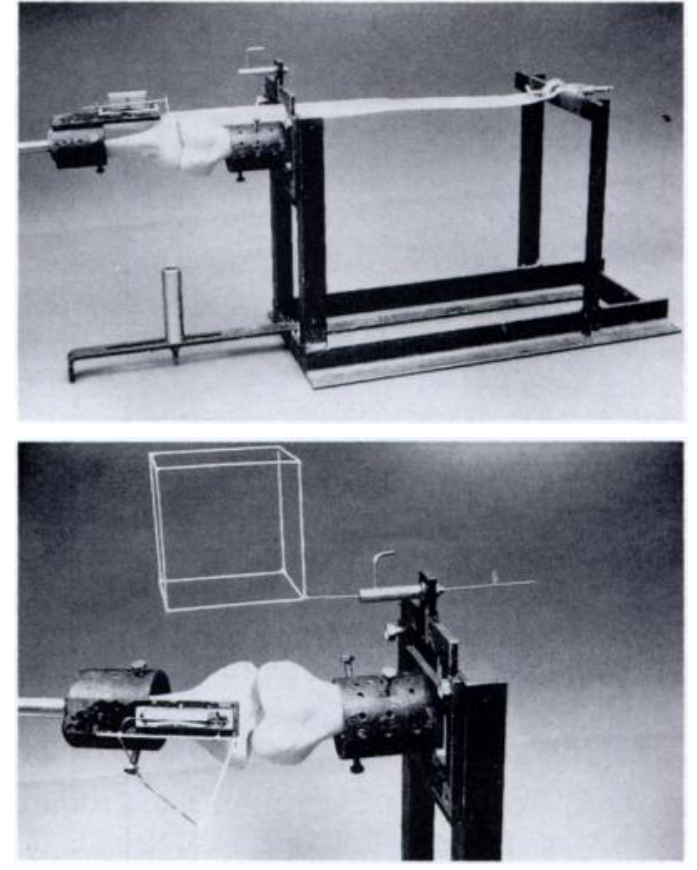

Fig. 2

Fig. 3

taking another biplanar photograph. From these two sets of films, the movements of the tibia with respect to the femur were calculated.

The films obtained were magnified about 20 times with a projector, and relevant points were recorded with a digitiser. From a series of two-dimensional co-ordinates of these points, projected onto the two biplanar film planes, the three-dimensional movements of the bones were calculated using a microcomputer.

The data were initially obtained in the form of a series of three-dimensional co-ordinates of the tibia with respect to the femur. However, since the main interest of this study was in the rotation and translation of the tibia during knee movement, the following parameters are presented in this paper:

1) Changes in tibial rotation with flexion angle,

2) Changes in the position of the tibial plateau, with respect to the femur, under different conditions of loading and after division of various ligaments.

\section{RESULTS}

The following describes the rotation of the tibia and its translational movement with respect to the femur, as the knee is gradually flexed from the fully extended position, whilst maintaining a valgus torque. The data were obtained first, from knees in which the ligaments, the capsule and the (artificial) ITT were all intact, then after dividing one or more of the ligaments.

Normal knees with intact ligaments. In normal knees, the tibia rotated internally about $10^{\circ}$ as it was flexed. 
The rotation took place between $20^{\circ}$ and $90^{\circ}$ of flexion (Fig. 4).

Effect of anterior cruciate ligament division. When only the anterior cruciate ligament ( $A C L$ ) was divided, a pivot shift, identified by an increase in internal rotation and a sudden reduction of the tibia was observed between $20^{\circ}$ and $40^{\circ}$ of flexion in seven of the 20 knees examined (Fig. 5). In the other 13, no pivot shift was observed, but instead a gradual increase and then a gradual reduction in internal rotation occurred (Fig. 6).

A typical example of the change in position of the tibial plateau from its position prior to $\mathrm{ACL}$ division to that after division, at $25^{\circ}$ of flexion, is shown in Figure 7. As can be seen, the tibia rotated internally around an axis located at the site of the medial collateral ligament (MCL). Similar findings were observed in all the other 19 knees (both in those with positive and negative pivot shifts). The amount of internal rotation was between $5^{\circ}$ and $17^{\circ}$, which corresponded to an anterior displacement of the lateral tibial plateau of between $5 \mathrm{~mm}$ and $21 \mathrm{~mm}$. Effect of division of the lateral capsule and ITT. Section of the lateral capsule alone produced no significant change in tibial rotation in any of the three knees examined. When both the lateral capsule and the ITT were divided, a large increase in internal rotation of the tibia (between $10^{\circ}$ and $21^{\circ}$ ) was observed, at flexion angles between $20^{\circ}$ and $80^{\circ}$ (Fig. 8).

Effect of combined division of the ACL and the lateral structures. In two of the three knees, section of both the $\mathrm{ACL}$ and the lateral capsule produced no further change from that observed after a simple ACL division. There was a small increase in the tibial internal rotation (less than $3^{\circ}$ ), at flexion angles between $40^{\circ}$ and $90^{\circ}$, in the third knee. When the ACL, the lateral capsule and the ITT were all divided, the pivot shift was not seen in any of the six knees examined, but a large increase in internal rotation (between $10^{\circ}$ and $33^{\circ}$ ) was observed throughout the range of movement, except at full extension. In one knee, the pivot shift, which had been observed after the preceding division of the $A C L$, disappeared when the ITT was divided (Fig. 9).

Effect of other ligament sections. When only the posterior cruciate ligament (PCL) was divided, a small increase in internal rotation was observed, at flexion angles between $60^{\circ}$ and $90^{\circ}$, in two of the three knees. In the third, the increase was observed between full extension and $30^{\circ}$ flexion and again between $60^{\circ}$ and $90^{\circ}$ flexion. However, the magnitude of increase in internal rotation was much smaller than that observed after ACL division (less than $4^{\circ}$ ). When both cruciates were divided, a large increase in internal rotation at flexion angles between $10^{\circ}$ and $60^{\circ}$ was observed, and a small increase or none throughout the rest of the flexion range in all of eight knees. The flexion-rotation curve was similar to that observed after simple division of the ACL. A pivot shift occurred in one of the eight knees, and a gradual increase and a gradual reduction in the other seven.
When only the MCL was divided, there was diminished internal rotation throughout the whole range of movement except a full extension, in all three knees examined. When both the ACL and the MCL were divided, the pivot shift was not observed, but anterior translation of both the medial and the lateral tibial plateau occurred at flexion angles between $10^{\circ}$ and $60^{\circ}$ in all six.

\section{DISCUSSION}

Cause of the pivot shift. Several different causes for the pivot shift have been proposed: 1) solitary ACL injury (Fetto and Marshall 1979), 2) combined injury to the $\mathrm{ACL}$ and the lateral capsular structures (Losee, Johnson and Southwick 1978), and 3) solitary lateral structure injury (Hughston et al 1976a,b).

In these experiments, the pivot shift, identified by an increase in internal rotation and a sudden reduction, was observed only after ACL division. Solitary division of the PCL, the lateral capsule, the MCL or the ITT did not produce the pivot shift. Nor was the pivot shift observed when other ligaments were divided in addition to the ACL. We conclude that solitary ACL division is responsible for the pivot shift.

Since the lateral capsule is a thin structure located under the thick ITT, it cannot effectively constrain internal rotation of the tibia so long as the ITT is intact. The ITT constrains internal rotation of the tibia, but it can only do so at large angles of flexion. Since the pivot shift is a phenomenon characterised by an increase in internal rotation at small angles of flexion followed by a sudden reduction at a larger flexion angle, division of ITT cannot cause the pivot shift. After a combined division of ACL and ITT, the tibia rotated internally throughout the range of flexion, and the pivot shift therefore disappeared. The internally rotated tibia, at about $30^{\circ}$ of flexion, remained rotated and did not relocate as flexion increased. A similar phenomenon was observed clinically by Losee (1983), who found that the pivot shift disappeared when the ITT was detached from Gerdy's tubercle during an ITT transfer operation.

Axis of the pivot shift. The axis of the pivot shift has been thought to be at the PCL (Hughston et al 1976a,b). However, from the results of this study, the axis of the pivot shift is, in fact, located about the MCL. This is explained as follows. When a valgus torque is applied to the tibia, the movements of the medial side of the tibia are limited by the tension in the MCL. Since the articular surface of the lateral tibial plateau is inclined downwards and backwards (Higgins 1896), a compressive force on the lateral tibial plateau produces a force component in the anterior direction (Fig. 10). Therefore, when a valgus torque is applied, the lateral tibial plateau is pushed forwards. Since the medial plateau is fixed by the tight MCL, internal rotation of the tibia results (Fig. 10). This torque is mainly resisted by the ACL at about $30^{\circ}$ of 


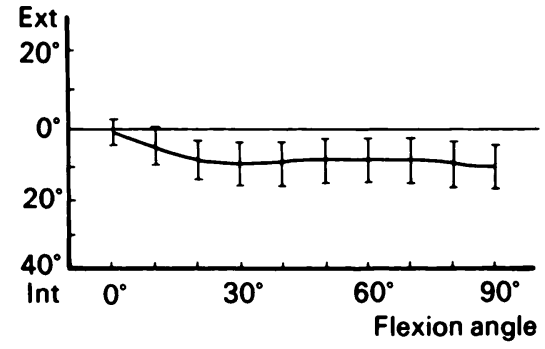

Fig. 4

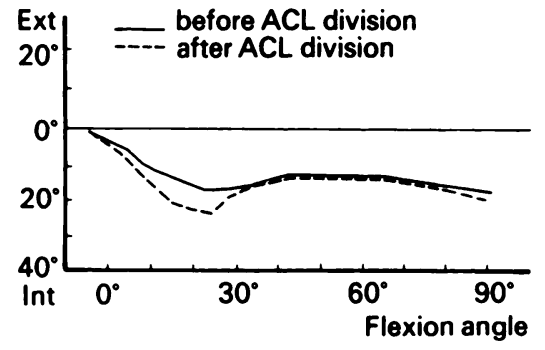

Fig. 5

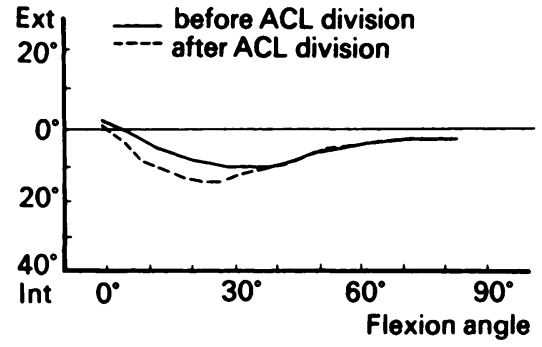

Fig. 6

Figure 4 - Tibial rotation under valgus torque in a normal cadaveric knee. Figure 5 - Tibial rotation under valgus torque after ACL division (positive pivot shift). Figure 6 - Tibial rotation under valgus torque after ACL division (negative pivot shift).

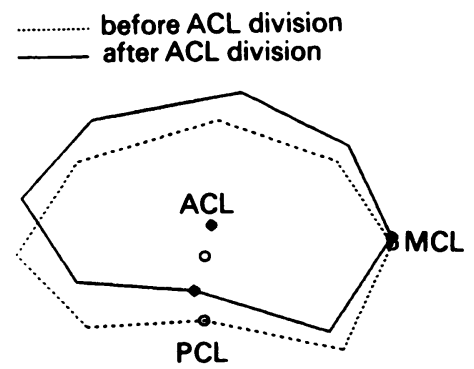

Fig. 7

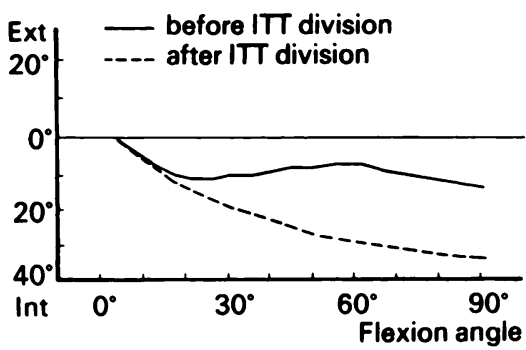

Fig. 8

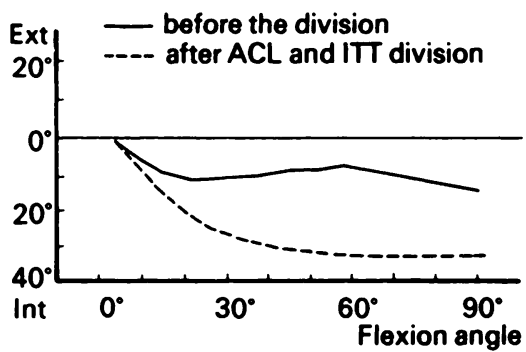

Fig. 9

Figure 7 - Change in position of the tibial plateau after division of the ACL at $25^{\circ}$ of flexion. Figure 8 - Tibial rotation under valgus torque after division of the lateral capsule and the iliotibial band. Figure 9 - Tibial rotation under valgus torque after division of the anterior cruciate, the lateral capsule, and the iliotibial band.
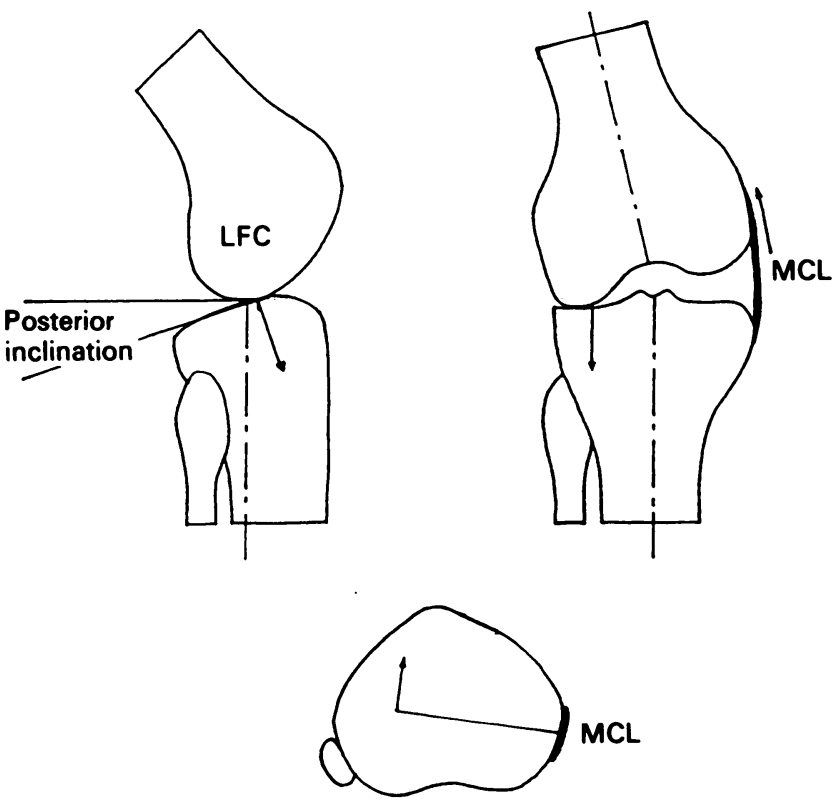

Fig. 10

Effect of the valgus torque on the knee: A compressive force on the lateral tibial plateau and a tensile force in the MCL, result in an internal rotatory torque about the MCL. LFC = lateral femoral condyle. flexion and therefore the tibia rotates internally about the $\mathrm{MCL}$, in that degree of flexion, when the ACL is absent.

Because the PCL was thought to be the axis of the rotation, it was believed that rotation after injury to both cruciates was different from rotation after a solitary injury to the ACL (Hughston et al 1976a,b). However, from the results of this study, combined division of both cruciates produced a pattern of tibial rotation similar to that seen after division of the ACL alone. This is because the axis of the pivot shift is, in fact, about the MCL. Division of the PCL has little effect upon tibial movement because the ligament is slack when the tibia is in internal rotation about the MCL.

The sudden movement of the pivot shift. Two different explanations for the sudden movement in the pivot shift have been proposed.

1) During movements of the knee, the ITT slides backward during flexion and forward during extension. When the ITT passes the centre of the flexion-extension movement, the function of the ITT is suddenly changed from being an extensor (pulling the lateral tibial plateau anteriorly) to becoming a flexor (pulling it posteriorly). This sudden change in the direction of pull of the ITT 
causes the sudden movement of the knee (Jakob, Hassler and Staeubli 1981).

2) The articular surface of the lateral tibial plateau is convex. When the joint is under a valgus torque, a compressive force acts between the lateral articulating surfaces. As the lateral femoral condyle rolls backward and forward over the tibial plateau during flexionextension movements, it has to pass over the apex of the convexity. When this point is reached (the top of the hill) there is a sudden movement as the femur slides down the other side. This sudden movement will be manifest as a subluxation or reduction, depending on whether the knee is being flexed or extended (Slocum et al 1976). knees or in them all if valgus torque of a different magnitude had been used.

One explanation for a negative pivot shift test in a knee with a divided ACL may be found in the geometry of the lateral tibial plateau. If this was flat or had a less pronounced convexity than usual, a gradual increase in rotation would result rather than a sudden jerk.

However, the important fact is that an increase in internal rotation occurred after division of the ACL alone in all knees examined, whether or not relocation of the internally rotated tibia occurred suddenly or gradually. In the clinical examination of patients, examiners can (and do) apply a rotatory torque or an anterior force to
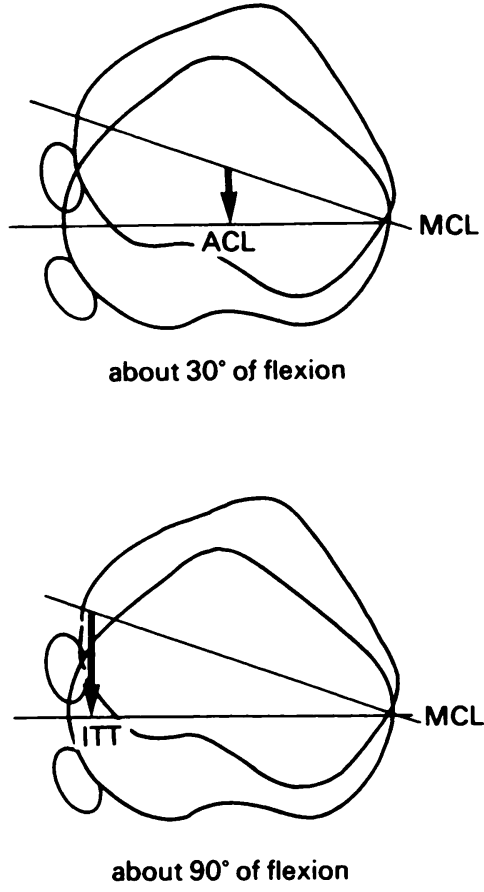

Fig. 11

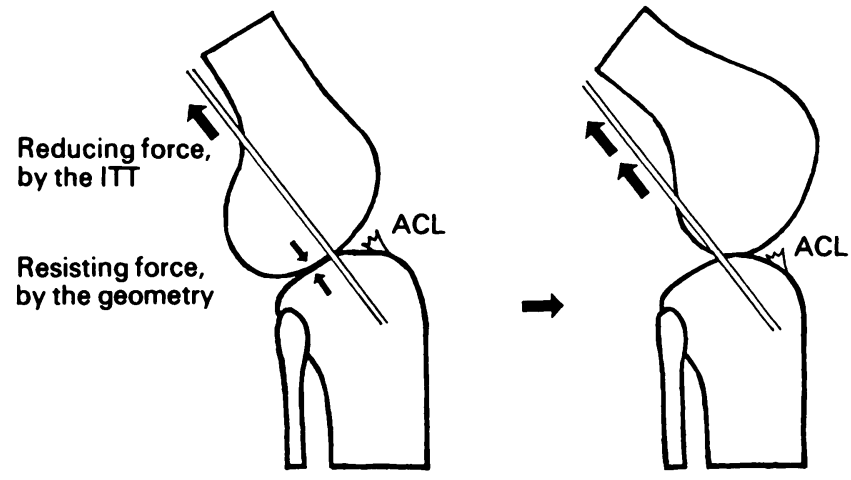

Fig. 12

Figure 11 - Constraints on internal rotation of the tibia about the MCL: ACL at about $30^{\circ}$ of flexion and ITT at flexion angles greater than $30^{\circ}$. Figure 12 - Mechanism of the pivot shift: the anteriorly subluxed lateral tibial plateau relocates suddenly, when a reducing force applied by the ITT becomes greater than the resistance exerted because of the geometry of the condyles.

In our experiments the ITT did not, in fact, act as an extensor to pull the tibia anteriorly with the knee at the nearly extended position. Furthermore, the amount of anterior translation of the lateral tibial plateau observed in the pivot shift was large (up to $21 \mathrm{~mm}$ ), quite sufficient for the lateral femoral condyle to move over the posterior edge of the lateral tibial plateau, as described by Maquet, Van De Berg and Simonet (1975). Therefore, the results of the present study support the second explanation, namely, that the sudden movement is caused by the interaction between the compressive force and the geometry of the lateral tibial plateau and femoral condyle. Anomalies. In this study, the pivot shift did not occur in every knee after severing the ACL. The magnitude of the valgus torque applied to the tibia was the same in all of them, to standardise the loading conditions, and it is possible that the pivot shift might have occurred in more the tibia, in addition to the valgus torque, throughout the pivot shift test or at the critical angle of flexion, and this may increase the percentage of positive results.

Mechanism of the pivot shift. The mechanism of the pivot shift can, therefore, be described as follows:

A valgus torque on the knee produces a tensile load along the MCL and a compressive one on the lateral side.

When the knee, subjected to a valgus torque, is flexed from the position of full extension, the tibia experiences an anterior force, due to the inclination of its lateral plateau.

When intact, the ACL resists this force and prevents the tibia from subluxing anteriorly (Fig. 11).

In an ACL-deficient knee, the lateral tibial plateau subluxes anteriorly, and the tibia rotates internally about the tight MCL. This occurs usually at flexion angles between $20^{\circ}$ and $40^{\circ}$. 
As the knee is flexed further, the tension in an intact ITT, acting with the geometry of the lateral tibial plateau, relocates the tibia (Fig. 12). If the ITT is severed, the tibia remains internally rotated.

\section{CONCLUSIONS}

1. The pivot shift phenomenon is a sudden subluxation of the tibia in a normal position, or the relocation of the internally rotated tibia about the MCL, when the knee is moved whilst applying and maintaining valgus torque on it.

2. Absence of the ACL is responsible for the anterior subluxation of the lateral tibial plateau, whilst the action of the ITT is responsible for the relocation of the subluxed tibia.

3. The sudden movement of the pivot shift occurs as a result of a complex interaction between the geometry (namely the convexity) of both the lateral articulating surfaces of the knee and the compressive force acting on the lateral side of the knee resulting from the valgus torque.

4. The absence of the sudden movement observed in some of the specimens tested is likely to be due to either a flat or less convex lateral tibial plateau, or to differences in the magnitude of valgus and/or internal torques on the tibia needed to produce the sudden movement commonly associated with the pivot shift phenomenon.

This work was carried out at the Rheumatology and Rehabilitation Unit, University of Leeds, under the supervision of Dr Bahaa Seedhom and Professor Verna Wright.

Thanks are due to Mr Michael Pullan and Mr Bryan Whitham for their technical assistance throughout the project, and to Professor Yabe, Professor Iseki and Dr Fujikawa, Keio University, for their support and encouragement of this project.

No benefits in any form have been received or will be received from a commercial party related directly or indirectly to the subject of this article.

\section{REFERENCES}

Fetto JF, Marshall JL. Injury to the anterior cruciate ligament producing the pivot-shift sign. J Bone Joint Surg [Am] 1979; 61A:710-4.

Galway RD, Beaupré A, Macintosh DL. Pivot shift: a clinical sign of symptomatic anterior cruciate ligament insufficiency. $J$ Bone Joint Surg [Br] 1972; 54-B: 763-4.

Higgins $\mathbf{H}$. The geniculate articular surfaces of the femur and tibia. $J$ Anat Physiol 1896; 30:292-5.

Hughston JC, Andrews JR, Cross MJ, Moschi A. Classification of knee ligament instabilities. Part I. The medial compartment and cruciate ligaments. J Bone Joint Surg [ Am] 1976a ; 58-A :159-72.

Hughston JC, Andrews JR, Cross MJ, Moschi A. Classification of knee ligament instabilities. Part II. The lateral compartment. J Bone Joint Surg [Am] 1976b; 58-A :173-9.

Jakob RP, Hassler H,Staeubli H-U. Observations on rotatory instability of the lateral compartment of the knee: experimental studies on the functional anatomy and the pathomechanism of the true and the reversed pivot shift sign. Acta Orthop Scand 1981; 52 Suppl 191.

Lipke JM, Janecki CJ, Nelson CL, et al. The role of incompetence of the anterior cruciate and lateral ligaments in anterolateral and anteromedial instability: a biomechanical study of cadaver knees. J Bone Joint Surg [ Am] 1981; 63-A :954-60.

Losee RE. Concepts of the pivot shift. Clin Orthop 1983; 172:45-51.

Losee RE, Johnson TR, Southwick WO. Anterior subluxation of the lateral tibial plateau: a diagnostic test and operative repair. $J$ Bone Joint Surg [Am] 1978; 60-A :1015-30.

Maquet PG, Van De Berg AJ, Simonet JC. Femorotibial weight-bearing areas. J Bone Joint Surg [ Am] 1975; 57-A :766-71.

Markolf KL, Graff-Radford A, Amstutz HC. In vivo knee stability: a quantitative assessment using an instrumented clinical testing apparatus. J Bone Joint Surg [Am] 1978; 60-A :664-74.

Matsumoto H. The pivot shift phenomenon: a biomechanical study. Thesis, Leeds 1987.

Shoemaker SC, Markolf KL. In vivo rotatory knee stability: ligamentous and muscular contributions. J Bone Joint Surg [Am] 1982; 64A :208-16.

Slocum DB, James SL, Larson RL, Singer KM. Clinical test for anterolateral rotary instability of the knee. Clin Orthop 1976; 118:63-9.

Wood MR, Dandy DJ. The cause and mechanics of the pivot shift phenomenon. J Bone Joint Surg [ Br] 1979; 61-B:519. 